\title{
Perspectives in urogynaecology: the surgical challenges ahead
}

\author{
P. Hinoul • H. A. M. Brölmann • J. P. Roovers
}

Received: 22 May 2007 / Accepted: 18 July 2007 / Published online: 16 August 2007

(C) Springer-Verlag 2007

\begin{abstract}
In this short review the new developments in urogynecology are addressed. The number of patients with pelvic organ prolaps (POP) is rising due to the ageing population. Between $4 \%$ and $50 \%$ of female patients complain of urinary incontinence. POP is related to (vaginal) parity. Because POP develops many years after vaginal birth trauma and not in all patients, a hereditary factor changing connective tissue function is suspected. In the diagnosis of pelvic floor disorders, imaging (ultrasound and MRI) are used more and more frequently. Pelvic floor surgery has high recurrence rates up to $30 \%$. Mesh augmented pelvic floor reconstruction is becoming more popular. Little randomised evidence is available to date, but slowly but surely more evidence accumulates supporting the use of mesh.
\end{abstract}

Keywords Pelvic organ prolapse - Urinary incontinence . Surgery · Medical treatment · Graft · Mesh · Physiotherapy · Medical treatment

\footnotetext{
P. Hinoul $(\bowtie)$

Department of Obstetrics and Gynaecology,

Ziekenhuis Oost-Limburg,

Schiepse Bos 6,

3600 Genk, Belgium

e-mail: piet.hinoul@skynet.be

H. A. M. Brölmann

Department of Obstetrics and Gynaecology,

VU University Medical Center Amsterdam,

Amsterdam, The Netherlands

J. P. Roovers

Department of Obstetrics and Gynaecology,

Academical Medical Center Amsterdam,

Amsterdam, The Netherlands
}

\section{Introduction}

Life expectancy has increased dramatically in the 20th century. Life expectancy at birth in the United States in 1900 was 47 years, whereas in 2000 life expectancy had increased to 77 years. Not only do patients live longer lives, most also live healthier lives allowing them to lead an active lifestyle for much longer than their ancestors. It is foreseen that over the next 30 years the number of patients requesting treatment because of pelvic organ prolapse (POP) and/or urinary inconcontinence (UI) will double [1]. The challenge for those involved in treating pelvic floor dysfunction is tremendous; more people demand surgery restoring the pelvic floor function and expect an assured outcome at an affordable cost. This paper wishes to address several issues that will occupy the field of urogynaecology in the near future.

\section{Etiology}

POP occurs in no less than $50 \%$ of patients above the age of 50. Age, pregnancy, vaginal delivery, menopause, smoking, and obesity have all been established as significant risk factors for POP. Racial differences are striking: European and Hispanic women are more at risk than African or Asian women to develop POP. Between 4\% and $50 \%$ of patients complain about urinary incontinence.

A risk factor that will attract a lot of attention in the next decade involves molecular alterations that alter the collagen composition. That molecular alterations rather than the trauma itself are important in the pathophysiology [2] of POP is plausible because the onset, in most cases, occurs decades after the inciting pelvic trauma. 
There is a clear relation between a reduced content of collagen of good quality and the presence of POP and/or UI. This is most likely due to an increased collagen degradation of freshly produced collagen, as production does not appear to be altered [3, 4]. A genetic or molecular predisposition that triggers this breakdown seems a plausible hypothesis as several studies have already confirmed maternal and paternal inheritance of POP and UI. Identifying these high risk genes in the future will allow identification of patients at risk. These could subsequently be targeted for counseling and preventive measures intended to prevent damage to the pelvic floor muscles and innervation. Development of new types of therapy to prevent or reverse the pathological breakdown of the supporting tissues can then be addressed.

\section{Diagnosis}

In the near future we expect an increasing role of imaging and a decreasing role of urodynamics in the diagnostic work-up of patients with POP and/or UI.

\section{Imaging}

Imaging studies investigating the association between pelvic floor measurements have demonstrated that a deep pouch of Douglas, a short perineal body, a wide levator hiatus, and a wide genital hiatus are related to the development of POP. An increasing knowledge of anatomy and the ongoing technical development of ultrasound and magnetic resonance imaging will certainly lead to an improved understanding of the biomechanics of the pelvic floor. These noninvasive tools can now be used to prospectively assess the pelvic floor status in prospective studies with long-term follow-up. Data derived from these studies are likely to shed new insights in the development of POP and UI. We expect that in the short term, improved imaging will reveal the pathogenesis of POP by acute pelvic floor trauma (e.g. delivery) and in the longer term by certain chronic diseases like obesity and obstructive lung disease.

\section{Urodynamic investigation}

The role of urodynamics remains controversial as sensitivity is rather low: urinary incontinence (UI) can not be confirmed during the urodynamic investigation in $25-30 \%$ of cases. It is demonstrated [5] that women with urodynamic stress incontinence can be correctly identified from clinical history alone with a sensitivity of $0.92(95 \%$ C.I.: $0.91-0.93)$ and specificity of $0.56(0.53-0.60)$. A clinical history for the diagnosis of detrusor overactivity (DO) was found to be $0.61(0.57-0.65)$ sensitive and $0.87(0.85-0.89)$ specific. Within secondary care, imaging of leakage by ultrasound was found to be effective in the diagnosis of USI in women with a sensitivity of 0.89 (0.84-0.93) and specificity of $0.82(0.73-0.89)$ [5]. It remains to be researched whether the addition of a full multichannel urodynamic investigation adds to the final outcome of the treatment of the incontinent female patient. As with imaging techniques it is a fair assumption that urodynamic techniques in the future will also be able to rely on noninvasive tools such as ultrasound and near infrared spectrophotometry.

\section{Treatment of POP and UI}

Pelvic floor muscle training (PFMT)

PFMT appears to be an effective treatment for adult women with stress or mixed incontinence and/or fecal incontinence. Although the anatomical abnormalities in patients with genital prolapse will not be corrected, pelvic physiotherapy may, in these patients, slow the progression, relieve functional symptoms, and improve the result of surgical correction. It is the role of the urogynaecologists to increase awareness and uptake of PFMT in the general population of this simple prophylactic and therapeutic measure, preferably from a young age onward. This could be optimized by offering physiotherapy before and after delivery or prolapse surgery.

\section{Oral treatment of overactive bladder}

Antimuscarinic agents are considered the standard of care for the treatment of overactive bladder (OAB) symptoms. Five different muscarinic (M) receptor types (M1-M5) have been identified, which vary in distribution throughout the body. In the bladder the predominant receptors are the M2 and M3 subtypes. Although the M2 subtype is the most abundant receptor in the bladder, it seems that the M3 receptor subtype is most directly responsible for the symptoms of $\mathrm{OAB}$ because it mediates detrusor contractility. The significant incidence of anticholinergic side effects is a major cause of noncompliance and discontinuation of treatment. Further research to target the M3 bladder receptor more specifically or different drug delivery systems are expected to increase current efficiency and compliance.

Surgery for urinary stress incontinence

Surgery for the treatment of UI has dramatically changed since the introduction of the tension free vaginal tape 
(TVT) developed by Ulmsten and Petros in the mid 1990 s. With continence rates as high as $81 \%$ after 91 months and level 1 evidence that cost-effectiveness of TVT is superior to Burch colposuspension, it is obvious that the midurethral sling has become the preferred management option for the treatment of urodynamic stress incontinence. In 2001 the transobturator (TOT) approach was introduced by Delorme as an alternative midurethral tape-suspension in order to avoid passing the retropubic space. Comparative studies have suggested that, as compared to TVT, TOT (TVT-O) has a lower risk of postoperative bladder retention and overactive bladder symptoms. Whether this holds true in randomised trials with long term follow-up has yet to be established. Recently, mini tapes like TVT-S (secure) and MiniArc have been introduced. These latest generation midurethral tape-suspensions no longer traverse the obturator foramen but anchor into the obturator internus muscle, thus hypothetically reducing the risk of damage to the obturator vessels and nerves and decreasing pain due to perforation of the adductor muscles.

Apart from the evolution of the mid-urethral tape, stem cell therapy is a very promising new therapy of stress incontinence. It is encouraging that in a rat model, restoration of functional motor units could be demonstrated by inserting muscle precursor cell autografts. At the moment, trials in humans are ongoing in which autologous fibroblasts are injected around the urethra that differentiate into myoblasts. The stemcells are injected into the sphincter under ultrasound guidance [6]. Preliminary studies of this new therapy seem promising. Stemcell research is still in its very infancy but these data, as well as publications on tissue engineering of bladder tissue in patients needing a cystoplasty, prove that cell regeneration therapy may not be that far off [7]. A role for regenerative therapy may exist in the immediate postpartum period, with injection of autologous (muscle and maybe even nerve) cells in the urethra, the anal sphincter, and/or the levator muscle.

\section{Surgery for pelvic organ prolapse (POP)}

In the next few decades, two ongoing debates related to prolapse surgery have to be resolved: (1) What is the exact role of meshes in prolapse surgery? and (2) Should prolapse surgery and stress incontinence surgery be combined in patients who have a combination of prolapse and evident or occult stress incontinence?

\section{Role of meshes in prolapse surgery}

In pursuit of the success of the midurethral polypropylene sling, and analogous to the inguinal hernia mesh repairs, urogynaecologists eagerly introduced synthetic meshes during surgical correction of POP. Overall a failure rate for traditional surgery of $30 \%$ is quoted. Most success rates in mesh surgery are estimated to be around $90 \%$. Very few patients, thus far, have been randomised to compare mesh versus no mesh; however, those few studies confirm the improved outcome with the use of mesh when treating the anterior compartment. There seems to be no statistical or clinically significant improvement when a mesh is used to treat posterior compartment prolapse. Prolapse of the middle compartiment can be treated with equal effectivity via the vaginal route by means of the sacrospinous ligament fixation or abdominally by sacrocolpopexy. The vaginal route however has a poor long term cure rate for the anterior compartiment. Recently, needle suspension techniques have been introduced that fix the mesh, posteriorly, through the levatorplate or through the sacrospinous ligament near the ischial spine, and fix an anterior mesh through the foramen obturatorius, with the top of the anterior mesh also approaching the ischial spine. These meshes truly reconstruct the lateral "level 2" suspension, the attachment of the vagina to the arcus tendineus ('white line') as described by deLancey. Randomised controlled trials comparing these new vaginal needle suspension techniques and the abdominal sacrocolpopexy are under way. A lot is to be expected from progress that is made in mesh technology. As a higher anatomical and functional success rate is overshadowed by erosionrelated morbidity due to the use of foreign material into the vagina, research is focusing on minimizing the foreign body (fibrotic) reaction without compromising the graft's strength.

\section{Should prolapse surgery and stress incontinence surgery be combined?}

In the surgical treatment of POP a lot of questions remain unanswered with regards to the sequence of therapeutic interventions that need to be undertaken to restore pelvic floor function. As symptoms often do not correlate with the grade of the prolapse, it is not always clear which compartment should be reconstructed. Also, when one compartment is restored, the newly freed space or the alteration of the vaginal axis predisposes the other compartment to develop prolapse.

A similar problem is the occurrence of de novo stress incontinence that affects approximately one in ten women undergoing prolapse repair. One could attempt to prevent de novo stress incontinence by combining prolapse and stress incontinence surgery. However, several observational studies concluded that this combination increased the risk of postoperative bladder retention and overactive 
bladder symptoms. The results of these observational studies were in contrast with the results of a recently published randomised trial comparing sacrocolpopexy with and without concomitant burch colposuspension [8]. The conclusion is that even in women without stress incontinence, sacrocolpopexy should be combined with Burch colposuspension to reduce postoperative symptoms of stress incontinence without increasing other lower urinary tract symptoms. Unfortunately, the authors decided to report on short term follow-up ( 3 months), and the quality of life related to overactive bladder symptoms was not addressed.

Furthermore, the combination of abdominal prolapse surgery and Burch colposuspension does not reflect the majority of todays prolapse surgery where the debate is focussed on combining vaginal prolapse surgery with a midurethral sling procedure. The advantage of combining the procedures is a decreased risk of postoperative stress incontinence, but one could also prefer a strategy where the prolapse is corrected first and the patient is informed that there is a chance that secondary stress incontinence surgery may be necessary. A multi-center randomised trial comparing these two strategies is in preparation.

\section{References}

1. Luber KM, Boero S, Choe JY (2001) The demographics of pelvic floor disorders: current observations and future projections. Am J Obstet Gynecol 184:1496-1501

2. Jack GS, Nikolova G, Vilain E, Raz S, Rodiquez LV (2006) Familial transmission of genitovaginal prolapse. Int Urogynecol J 17:498-501

3. Soderberg MW, Falconer C, Bystrom B, Malmstrom A, Ekman G (2004) Young women with genital prolapse have a low collagen concentration. Acta Obstet Gynecol Scand 83:1193-1198

4. Jackson SR, Avery NC, Tarlton JF, Eckford SD, Abrams P, Bailey AJ (1996) Changes in metabolism of collagen in genitourinary prolapse. Lancet 347:1658-1661

5. Martin JL, Williams KS, Abrams KR, Turner DA, Sutton AJ, Chapple C et al (2006) Systematic review and evaluation of methods of assessing urinary incontinence. Health Technol Assess 10:1-9

6. Strasser H, Berjukow S, Marksteiner R, Margreiter E, Hering S, Bartsch G et al (2004) Stem cell therapy for urinary stress incontinence. Exp Gerontol 39:1259-1265

7. Atala A, Bauer SB, Soker S, Yoo JJ, Retik AB (2006) Tissueengineered autologous bladders for patients needing cystoplasty. Lancet 367:1241-1246

8. Brubaker L, Cundiff GW, Fine P, Nygaard I, Richter HE, Visco AG et al (2006) Abdominal sacrocolpopexy with Burch colposuspension to reduce urinary stress incontinence. $\mathrm{N}$ Engl $\mathrm{J}$ Med $354: 1557-1566$ 